\title{
Quantum Black Holes. Black Hole Temperature without a Black Hole.
}

\author{
Victor Berezin* \\ Institute for Nuclear Research \\ Russian Academy of Sciences \\ Moscow, 117312, 60-October Anniversary pr.,7-a \\ E-mail: berezinems2.inr.ac.ru
}

The model is constructed, some features of which comes from quantum thin dust shells and is, in fact, an extension of the "no hair" property of classical black hole on a quantum level. It appears that the proposed classical analog of quantum black hole is heated, the temperature being exactly the Hawking's temperature.

Black Holes in General Relativity and String Theory August 24-30 2008

Veli LoÅainj, Croatia

${ }^{*}$ Speaker. 


\section{Quantum shells.}

In series of papers [1] a quantum mechanics for spherically symmetric thin dust shells has been developed. Here we write out only some necessary results. It was shown that the WheelerDeWitt equation is reduced in this case to the following stationary Schroedinger equation in finite differences:

$$
\Psi\left(m, m_{\text {in }}, S+i \zeta\right)+\Psi\left(m, m_{\text {in }}, S-i \zeta\right)=\frac{F_{\text {in }}+F_{\text {out }}-\frac{M^{2}}{4 m^{2} S}}{\sqrt{F_{\text {in }}} \sqrt{F_{\text {out }}}} \Psi\left(m, m_{\text {in }}, S\right),
$$

where $m=m_{\text {out }}=m_{\text {tot }}$ - the total mass of the system, $m_{\text {in }}$ - the Schwarzschild mass inside, $M$ is the bare mass of the shell, $S=\frac{R^{2}}{4 G^{2} m^{2}}\left(R\right.$ - radius, $G$ - gravitational constant), $F=1-\frac{2 G m}{R}, \zeta=\frac{m_{P l}^{2}}{2 m^{2}}$ $\left(m_{P l}=\sqrt{\frac{\hbar c}{G}}\right.$ is the Planckian mass and we use units with $\hbar=c=k=1, \hbar$ - Planck constant, $c$ - speed of light, $k$ - Bolzmann constant). By investigation of wave functions in the vicinity of singular points (infinities and singularities) and around the branching points (apparent horizons) the following discrete mass spectrum for bound states was found $\left(\Delta m=m_{\text {out }}-m_{\text {in }}\right)$ :

$$
\begin{aligned}
\frac{2(\Delta m)^{2}-M^{2}}{\sqrt{M^{2}-(\Delta m)^{2}}} & =\frac{2 m_{P l}^{2}}{\Delta m+m_{i n}} n, \\
M^{2}-(\Delta m)^{2} & =2(1+2 p) m_{P l}^{2},
\end{aligned}
$$

where $n$ and $p \geq 0$ are integers. The appearance of two quantum numbers instead of one in conventional quantum mechanics is due to the nontrivial causal structure of the complete Schwarzschild manifold.

The above spectrum is not universal in the sense that the corresponding wave functions form a two-parameter family $\Psi_{n, p}$. But for the quantum Schwarzschild black hole we expect a oneparameter family of solutions, because quantum black holes should not have "no hairs", otherwise there will be no smooth classical limit. This means that our spectrum is not a quantum black hole spectrum, and corresponding quantum shells do not collapse (like an electron in hydrogen atom). Physically, it is quite understandable, because the radiation was not included into consideration. The energy of radiation is also quantized, but, and this is crucial, the energies of quanta are not equal to the level splitting in the shell discrete spectrum, Eqn.(1.2). As a result, the quantum gravitational collapse proceeds via production new shells, thus increasing the inner mass $m_{i n}$ inside the primary shell. Such a process can go in many different ways, so, it is the quantum collapse that appears to be the origin of the black hole nonzero entropy. But how could quantum collapse be stopped? The natural limit is the transition from a black hole-like shell to a wormhole-like shell by crossing an Einstein-Rosen bridge, since such a transition requires (at least in a quasi-classical regime) insertion of infinitely large volume, which probability is, of course, zero. Computer simulations show that the process of quantum gravitational process stops when the principal quantum number becomes zero, $n=0$.

The point $n=0$ in our spectrum is very special. In this state the shell does not "feel" not only the outer region (what is natural for the spherically symmetric configuration), but it does not know anything about what is going on inside. It "feels" only itself. Such a situation reminds the "no hair" property of a classical black hole. Finally, when all the shells (both the primary one and newly 
produced) are in the corresponding states $n_{i}=0$, the whole system does not "remember" its own history. And it is this "no memory" state that can be called "the quantum black hole". Note, that the total masses of all the shells obey the relation

$$
\Delta m_{i}=\frac{1}{\sqrt{2}} M_{i}
$$

The subsequent quantum Hawking's evaporation can proceed via some collective excitations and formation, e.g., of a long chain of microscopic semi-closed worlds.

\section{Classical analog of quantum black hole.}

The final state of quantum gravitational collapse, the quantum black hole, can be viewed as some stationary matter distribution. Therefore, we may hope that for massive enough quantum black hole such a distribution is described approximately by a classical static spherically symmetric perfect fluid with energy density $\varepsilon$ and pressure $p$ obeying classical Einstein equations. This is what we call a classical analog of a quantum black hole. Of course, in such a case the corresponding classical distribution has to be very specific. To study its main features let us consider the situation in more details.

Any static spherically symmetric metric can be written in the form

$$
d s^{2}=e^{v} d t^{2}-e^{\lambda} d r^{2}-r^{2}\left(d \theta^{2}+\sin ^{2} \theta d \varphi^{2}\right) .
$$

Here $r$ is the radius of a sphere with the area $S=4 \pi r^{2}, v=v(r), \lambda=\lambda(r)$. The Einstein equations are (prime denotes differentiation in $r$ ):

$$
\begin{aligned}
-e^{-\lambda}\left(\frac{1}{r^{2}}-\frac{\lambda^{\prime}}{r}\right)+\frac{1}{r^{2}} & =8 \pi G \varepsilon, \\
-e^{-\lambda}\left(\frac{1}{r^{2}}+\frac{v^{\prime}}{r}\right)+\frac{1}{r^{2}} & =-8 \pi G p, \\
-\frac{1}{2}\left(v^{\prime \prime}+\frac{v^{\prime 2}}{2}+\frac{v^{\prime}-\lambda^{\prime}}{r}-\frac{v^{\prime} \lambda^{\prime}}{2}\right) & =-8 \pi G p .
\end{aligned}
$$

We see that there are three equations for four unknown functions of one variable, namely, $v(r), \lambda(r)$, $\varepsilon(r)$ and $p(r)$. But, even we would know an equation of state for our perfect fluid, $p=p(\varepsilon)$, the closed (formally) system of equations would have too many solutions. We need, therefore, some selection rules in order to single out the classical analog of quantum black hole. Surely, the "no hair" feature should be the main criterium. Thus, we have to adjust our previous definition of the "no memory" state to the case of a continuum matter distribution. For this, let us integrate the first of Eqns.(2.2):

$$
e^{-\lambda}=1-\frac{2 G m(r)}{r}
$$

where

$$
m(r)=4 \pi \int_{0}^{r} \varepsilon \tilde{r}^{2} d \tilde{r}
$$


is the mass function that must be identified with $m_{i n}$. Now, the "no memory" principle is readily formulated as the requirement, that $m(r)=a r^{2}$ [2], i.e.,

$$
e^{-\lambda}=1-2 G a=\text { const } \text {. }
$$

Note, that in static case, the inverse metric coefficient $e^{-\lambda}$ is an invariant which in the general spherically symmetric space-time reads as $\Delta=-e^{-\lambda}=g^{i k} R_{, i} R_{, k}$ and is nothing more but a squared normal vector to the surface of constant radius $R\left(x^{i}\right)=R(t, q)=$ const. We can also introduce a bare mass function $M(r)$ (the mass of the system inside a sphere of radius $r$ without the gravitational mass defect).

$$
M(r)=4 \pi \int_{0}^{r} \varepsilon(\tilde{r}) e^{\frac{\lambda}{2}}(\tilde{r}) \tilde{r}^{2} d \tilde{r}=\frac{a r}{\sqrt{1-2 G a}} .
$$

The remaining two equations (2.2) can now be solved for $p(r)$ and $e^{v}(r)$. The general solution is rather complex, but the correct non-relativistic limit for the pressure $p(r)$ (we are to reproduce the famous equation for hydrostatic equilibrium) has only the following one-parameter family:

$$
p(r)=\frac{b}{4 \pi r^{2}},
$$

where

$$
b=\frac{1}{G}(1-3 G a-\sqrt{1-2 G a} \sqrt{1-4 G a}) .
$$

We see that the solution exists only for $a \leq \frac{1}{4 G}$, then $b \leq a$. The physical meaning of these inequalities is that the speed of sound cannot exceed the speed of light, $v_{\text {sound }}^{2}=\frac{b}{a} \leq 1=c^{2}$, the equality being reached just for $a=b=\frac{1}{4 G}$. Finally, for the temporal metric coefficient $g_{00}=e^{v}$ we get:

$$
e^{v}=C_{0} r^{\frac{4 b}{a+b}}=C_{0} r^{2 G \frac{a+b}{1-2 G a}} .
$$

Thus, demanding the "no memory" feature and existence of the correct non-relativistic limit, we obtained the two-parameter family of static solutions. But we need a one-parameter family, so we have to continue our search. Let us investigate the obtained space-time manifolds more thoroughly, especially in the vicinity of the apparently singular point $r=0$, and calculate the corresponding curvature (Riemann's) tensor $R_{v \lambda \sigma}^{\mu}$. The nonzero components are

$$
\begin{aligned}
& R_{101}^{0}=2 \frac{b(a-b)}{(a+b)^{2}} \frac{1}{r^{2}} ; R_{202}^{0}=2 \frac{b}{a+b}(1-2 G a) ; R_{303}^{0}=R_{202}^{0} \sin ^{2} \theta ; \\
& R_{010}^{1}=2 C_{0}^{2} \frac{b(b-a)}{(a+b)^{2}}(1-2 G a) r^{\frac{2(b-a)}{a+b}} ; \\
& R_{020}^{2}=2 C_{0}^{2} \frac{b}{a+b}(1-2 G a) r^{\frac{2(b-a)}{a+b}} ; R_{323}^{2}=2 G a \sin ^{2} \theta ; \\
& R_{030}^{3}=2 C_{0}^{2} \frac{b}{a+b}(1-2 G a) r^{\frac{2(b-a)}{a+b}} ; R_{232}^{3}=2 G a .
\end{aligned}
$$

Evidently, for $b<a$ the Riemann tensor (2.10) is divergent at $r=0$, so, the corresponding spacetimes have the real singularity. But, if $a=b=\frac{1}{4 G}$ we are witnessing a miracle, the (before) diver- 
gent components become zero, and the remaining nonzero ones equal

$$
\begin{aligned}
& R_{202}^{0}=-(1-2 G a)=-\frac{1}{2}, \quad\left(R_{020}^{2}=\frac{1}{2} C_{0}^{2}\right) ; \\
& R_{303}^{0}=-(1-2 G a)=-\frac{1}{2}, \quad\left(R_{030}^{0}=\frac{1}{2} C_{0}^{2}\right) ; \\
& R_{323}^{2}=2 G a \sin ^{2} \theta=\frac{1}{2} \sin ^{2} \theta, \quad\left(R_{232}^{3}=\frac{1}{2}\right),
\end{aligned}
$$

and the only nonzero component of the Ricci tensor $R_{\mu v}\left(=R_{\mu \alpha \nu}^{\alpha}\right)$ equals to

$$
R_{00}=C_{0}^{2}
$$

Thus, demanding, in addition to the previous two very natural requirements, the third one (also natural), namely, the absence of the real singularity at $r=0$, we arrive at the following one-parameter family to the Einstein equations (2.2):

$$
\begin{aligned}
g_{00} & =e^{v}=C_{0}^{2} r^{2}, \\
g_{11} & =-e^{\lambda}=-\sqrt{2}, \\
\varepsilon & =p=\frac{1}{16 \pi G r^{2}} .
\end{aligned}
$$

So, the equation of state of our perfect fluid is the stiffest possible one. The constant of integration $C_{0}$ can be determined by matching the interior and exterior metrics at some boundary radius $r=r_{0}$. Let us suppose that for $r>r_{0}$ the space-time is empty, so, the interior should be matched to the Schwarzschild metric, labeled by the mass parameter $m$. Of course, to compensate the jump in the pressure $\Delta p\left(=p\left(r_{0}\right)=p_{0}\right)$ we must include in our model some surface tension $\Sigma$. It is easy to check, that

$$
\begin{aligned}
C_{0}^{2} & =\frac{1}{2 r_{0}^{2}} ; \Delta p=\frac{2 \Sigma}{\sqrt{2} r_{0}} ; \\
e^{v} & =\frac{1}{2}\left(\frac{r}{r_{0}}\right)^{2} ; p_{0}=\varepsilon_{0}=\frac{1}{16 \pi G r_{0}^{2}} ; \\
m & =m_{0}=\frac{r_{0}}{4 G} .
\end{aligned}
$$

Note, that the bare mass $M=\sqrt{2} m$, the relation is exactly the same as for the shell "no memory" state (1.3), and $r_{0}=4 G m_{0}$, so, the size of our analog of quantum black hole is twice as that of classical black hole. But how about the special point in our solution, $r=0$ ? It is not a trivial coordinate singularity, like in a three-dimensional spherically symmetric case, because

$$
d s^{2}(r=0)=0
$$

This looks rather like an event horizon. To clear the point we consider the radial geodesic motion in the space-time with the metric

$$
d s^{2}=\frac{1}{2}\left(\frac{r}{r_{0}}\right) d t^{2}-2 d r^{2}-r^{2}\left(d \theta^{2}+\sin ^{2} \theta d \varphi^{2}\right)
$$


The calculations are very simple, the falling bounded geodesics are described by the following function $r(t)$, or $r(\tau)$ for the proper time parameter $\tau$ :

$$
\begin{aligned}
r(\tau) & =\frac{1}{\sqrt{2}} \sqrt{2 r_{1}^{2}-\tau^{2}} \\
\tau & =\sqrt{2} r_{1} \tanh \frac{t}{2 r_{0}} \\
r(t) & =\frac{r_{1}}{\cosh \frac{t}{2 r_{0}}}
\end{aligned}
$$

where we put $r=r_{1}<r_{0}$ for $\tau=t=0\left(\frac{d r}{d \tau}\left(r_{1}\right)=\frac{d r}{d t}\left(r_{1}\right)=0\right)$. We see that the surface $r=0$, indeed, behaves like an event horizon (and, at the same time, a Killing horizon). Investigation of non-radial geodesics shows an infinite spiralling when approaching zero radius surface, thus confirming its horizon nature. Moreover, the two-dimensional part of the metric (2.16), i.e., $(t-r)$ surface, is locally flat, what can easily be proven by making the following coordinate transformation $(t, r) \rightarrow(\eta, x):$

$$
\eta=\sqrt{2} r \sinh \frac{t}{2 r_{0}}, x=\sqrt{2} r \cosh \frac{t}{2 r)} .
$$

This resembles the Rindler's transformation in two-dimensional flat Minkowski space-time.

\section{Rindler space-time.}

The Rindler space-time is obtained by transforming the two-dimensional Minkowski spacetime from the ordinary coordinates $(\eta, x)$ and metric $d s^{2}=d \eta^{2}-d x^{2}$ related to the set of inertial observers, to the so-called Rindler coordinates and metric

$$
\begin{aligned}
\eta= & \frac{1}{a} e^{a \xi} \sinh a \eta, \quad x= \pm \frac{1}{a} e^{a \xi} \cosh a \eta(x \geq 0) \\
& -\infty<t<\infty,-\infty<\xi<\infty \\
& d s^{2}=e^{2 a \xi}\left(d t^{2}-d \xi^{2}\right)
\end{aligned}
$$

Thus, the Rindler space-time is static and locally flat but differs from the two-dimensional Minkowski space-time globally, because it covers only one half of the latter and, in addition, possesses the event horizons at $\eta= \pm x(t= \pm \infty, \xi=$ const $)$. The Rindler observers $\xi=$ const undergo nonzero constant acceleration. The norm of the acceleration vector $a^{\mu}$ equals

$$
\alpha=\sqrt{\left|a^{\mu} a_{\mu}\right|}=a e^{-a \xi} .
$$

Existence of the horizons has a very important consequence. W.G.Unruh showed [3] that the quantum theory of scalar field in the Rindler space-time is, in fact, the finite temperature quantum field theory, and the value of the Unruh's temperature is

$$
T_{U}=\frac{a}{2 \pi}
$$

We see, that this temperature is proportional to the acceleration of the Rindler observer sitting at $\xi=0$ with $g_{00}=1$. But, all these observers are equivalent (we can always shift the spatial 
coordinate $\xi \rightarrow \xi-\xi_{0}$ ). The temperature value is not an invariant but it is a temporal component of a heat vector. This means that each observer measures the Unruh temperature when using its proper time $\tau(d s=d \tau)$. If the same observer uses the local clocks that show the local time $t\left(d s=\sqrt{g_{00}} d t\right)$, the local temperature measured by him equals

$$
T_{l o c}=\frac{T_{U}}{\sqrt{g_{00}}}=\frac{a}{2 \pi} e^{-a \xi}=\frac{\alpha}{2 \pi} .
$$

We know from the university course of thermodynamics (se, e.g., [3]) that the condition for thermal equilibrium in static space-times is $T_{l o c} \sqrt{g_{00}}=$ const. We can introduce an "apparent temperature" $T_{a p p}$ which is the local temperature of an observer sitting at $\xi=\xi_{2}$, "seen" by an observer at $\xi=\xi_{1}$ :

$$
T_{a p p}\left(\xi_{2}, \xi_{1}\right)=\frac{\sqrt{g_{00}(2)}}{\sqrt{g_{00}(1)}} T_{l o c}\left(\xi_{2}\right) .
$$

Then the condition for thermal equilibrium can be formulated as $T_{l o c}\left(\xi_{1}\right)=T_{a p p}\left(\xi_{2}\right)$ for all values $\xi=\xi_{2}$. Thus, the Rindler observers are in thermal equilibrium with each other. And the question arises: is the Rindler space-time unique in this sense? To answer it, consider some general twodimensional static space-time with a metric

$$
d s^{2}=e^{v} d t^{2}-d \rho^{2}=e^{v} d t^{2}-e^{\lambda} d q^{2} .
$$

Note, that in the Rindler case $\rho=\frac{1}{a} e^{a \xi}, e^{v}=\frac{\rho^{2}}{a^{2}}=g_{00}$. The static observer in the metric (3.6) undergoes a constant acceleration with the invariant $\alpha=\frac{1}{2}\left|\frac{d v}{d \rho}\right|=\frac{1}{2}\left|\frac{d v}{d q}\right| e^{-\frac{\lambda}{2}}$, and the (local) Rindler parameter $a(\rho)$, which is now called "the surface gravity $\kappa$ ", equals

$$
\kappa=\frac{1}{2}\left|\frac{d v}{d q}\right| e^{\frac{v-\lambda}{2}}=\frac{1}{2}\left|\frac{d v}{d \rho}\right| e^{\frac{v}{2}} .
$$

The thermal equilibrium condition requires $\kappa=$ const, therefore, $g_{00}=C \rho^{2}$, and this proves that the Rindler space-time is the only one which static observers are in the mutual thermal equilibrium.

\section{Topological temperature.}

The fact that an accelerated observer "sees" particles while moving in the empty (vacuum) Minkowski space-time, was known long ago to quantum field theorists. The physical reason for this phenomenon is obvious: particle creation is caused by the same forces that cause the particle detector's acceleration. From the quantum field theory point of view, the vacuum of an accelerated observer (=detector) is different from that of an inertial observer. And the empty space of the latter appeared filled with particles to the first one. Due to Unruh's discovery we know that the observer moving with constant acceleration detects particles with Planckian spectrum at the temperature proportional to the value of this acceleration. But, let us consider the following Gedankenexperiment. Two observers sitting in the rockets and bringing particle detectors with them, are moving inertially in the two-dimensional Minkowski space-time. At some definite moment they switch on the engines and start to move with equal constant accelerations. Surely, their detectors start to register particles. Because an amount of fuel in the rockets is finite, our observers will eventually become inertial again, and let the durations of their accelerated motion are different. Then, 
the number of detected particles will also be different and finite. Suppose, our observers are well educated and know about the Unruh effect, so, they are able to compare the Planckian spectrum with that obtained by them. Evidently, they will find the deviations from the thermal spectrum, and the longer their accelerations, the smaller will be these deviations. Thus, the appearance of the temperature in the Rindler space-time is a global effect - the acceleration should last infinitely both in the past and in the future.

Thermodynamically this can be understood as follows. The existence of the event horizons in the Rindler space-time prevents receiving any information from the remaining part of a manifold. And the observers can explain this loss of information by ascribing a nonzero entropy to the unseen part of a geodesically complete space-time. And the very appearance of the entropy and the static character of the Rindler space-time lead us to the notion of thermal equilibrium and, thus, to the notion of temperature. Therefore, we see that it is the event horizon that causes the spectrum of particles detected by the constantly accelerated observer to be the Planckian one. And the global character of the notion of event horizon is reflected in the global character of the Unruh's temperature.

It is amazing, but the Rindler temperature can be calculated without a thorough investigation of quantum field theory. For this we should make a Whick rotation to the imaginary Rindler time $\tau=i t$, then the Euclidean Rindler metric

$$
d l^{2}=a^{2} \rho^{2} d \tau^{2}+d \rho^{2}
$$

can be interpreted as that of a locally flat two-dimensional surface in polar coordinates (the polar angle is proportional to $\tau$ ) provided the imaginary time is periodic, the latter requirement is quite natural because in terms of Minkowskian time $\eta$ such a transition reads as $\eta=\frac{\rho}{a} \sinh ($ iat $)=i \frac{\rho}{a} \sin \tau$. The metric (4.1) describes, in general, the geometry of a cone embedded into the three-dimensional flat space with a conical singularity at $\rho=0$. But, if the period is $\tau=\frac{2 \pi}{a}$, the singularity disappears and we obtain the whole plane. In the finite temperature field theories the temperature is introduced as the inverse period of the imaginary time, and we see that in our case it is exactly the Unruh temperature (3.3)! We can call the temperature found in this way "the topological temperature", because the geometry in the vicinity of a single (but singular) point, determines the properties of the whole manifold.

\section{Black hole temperature with and without black holes.}

By the Einstein equivalence principle we can extend all we learned studying Rindler spacetimes, to the static gravitational fields, especially to the static spherically symmetric manifolds, because after fixing spherical angles $\theta$ and $\varphi$ they become, in fact, the two-dimensional surfaces. Of course, in general these surfaces are curved, the equivalence principle holds only locally and, therefore, static observers sitting at different values of radius will "feel" not only different temperatures, but by no means they will be in thermal equilibrium with each other. Such a temperature is observer dependent and cannot be considered as an intrinsic property of a given space-time. But, we saw that the Rindler space-time possesses the event horizons what is crucial for ascribing and entropy and temperature to the manifold itself (or to its part). 
So, we are looking for some examples of spherically symmetric static manifolds which possess the event horizons. And, of course, these are the well-known Schwarzschild and ReissnerNordstrom space-times outside the corresponding black holes. In both cases the metric can be written in the form

$$
\begin{aligned}
d s^{2}= & F d t^{2}-\frac{1}{F} d r^{2}-r^{2}\left(d \vartheta^{2}+\sin ^{2} \vartheta d \varphi^{2}\right), \\
& F=1-\frac{2 G m}{r}+\frac{G e^{2}}{r^{2}}=\left(1-\frac{r_{+}}{r}\right)\left(1-\frac{r_{-}}{r}\right),
\end{aligned}
$$

where $m$ is the mass, $e$ is the electric charge of Reissner-Nordstrom black hole, $r_{ \pm}=G m \pm$ $\sqrt{G^{2} m^{2}-G e^{2}}$ are, respectively, positions of the event and Cauchy horizons, and for $e=0$ the above relations become the parameters of a Schwarzschild black hole. After the Whick rotation to the imaginary time $\tau$ the Euclidean two-dimensional surface in the vicinity of the event horizon $r_{+}$ is described by the metric

$$
\begin{array}{r}
d l^{2}=\frac{\left(r_{+}-r_{-}\right)^{2}}{4 r_{+}^{2}} \rho^{2} d t^{2}+d \rho^{2}, \\
\rho=\frac{2 r_{+}}{\sqrt{r_{+}-r_{-}}} \sqrt{r_{+} r_{-}} \ll r_{+} .
\end{array}
$$

Thus, the topological temperature of the Reissner-Nordstrom black hole is

$$
T_{\text {top }}=\frac{r_{+}-r_{-}}{4 \pi r_{+}^{2}}
$$

This value equals $\frac{\kappa_{B H}}{2 \pi}$, where $\kappa_{B H}$ is the surface gravity calculated at the black hole event horizon that enters the first law of thermodynamics for black holes [5] [6]. And the topological temperature $T_{\text {top }}$ in the case of Schwarzschild black hole is exactly the famous Hawking temperature

$$
T_{H}=\frac{1}{8 \pi G m}
$$

obtained by explicit construction of the massless scalar quantum field theory on the Schwarzschild curved background with specific boundary conditions at the event horizon [7]. The temperature $T_{H}$ is the temperature of the Schwarzschild black hole seen by the distant static observer (at spatial infinity) for whom $g_{00}=1$. Other static observers sitting at the radius $r$, see the black hole apparent temperature $T_{a p p}=\frac{T_{H}}{\sqrt{g_{00}(r)}}$. This apparent temperature does not coincide with their local Rindler temperature $T_{l o c}=\frac{\kappa(r)}{2 \pi \sqrt{g_{00}}}$ what indicates that the static black hole is not in thermal equilibrium with the surrounding vacuum space-time (i.e., there is no heat bath) and, in fact, evaporates.

At last, let us turn to our model, the classical analog of quantum black hole. The metric (2.16) for the internal part of the model ( $r \leq r_{0}, \theta, \phi=$ const) is already in the Rindler form, if we put $\rho=\sqrt{2} r$. So, all static observers are in thermal equilibrium, and calculation of the topological temperature is straightforward. But there is one subtle and important point, in our case the horizon is at zero radius, $r=0$, which is at the same time the coordinate singularity of the whole fourdimensional space-time, therefore, we are not allowed to confine ourselves to the two-dimensional $(t-r)$-section. And after the Whick rotation we have to consider the four-dimensional Euclidean 
space-time where, in addition to the periodic time $\tau$, there are two spherical angles, $\theta$ and $\phi$. The regularity condition requires the period $2 \pi$ for only one of the angles, all other should have the period $\pi$. Since the period $2 \pi$ is already reserved for the azimuthal angle $\phi$, the topological temperature in our model equals

$$
T_{\text {top }}=\frac{1}{2 \pi r_{0}} \text {. }
$$

Remembering now that $r_{0}=4 G m_{0}$, we get for the classical analog of quantum black hole

$$
T_{B H}=\frac{1}{8 \pi G m_{0}}
$$

and this is exactly the Hawking temperature!

\section{Acknowledgements.}

The author is indebted to the Russian Foundation for Fundamental Researches for financial support (Grant N 06-02-16-342 a).

\section{References}

[1] V.A.Berezin, N.G.Kozimirov, V.A.Kuzmin, I.I.Tkachev, Phys.Lett., B212 (1988) 415. V.A.Berezin, Phys.Lett,, B241 (1990) 194; Phys.Rev., D55 (1997) 2139. V.A.Berezin, A.M.Boyarsky, A.Yu.Neronov, Phys.Rev., D57 (1998) 1118; Phys.Lett., B455 (1999) 109.

[2] V.A.Berezin, Nucl.Phys., B661 (2003) 409.

[3] W.G.Unruh, Phys.Rev., D14 (1976) 870.

[4] L.D.Landau, E.M.Lifshits, "Theoretical Physics", Vol.5. "Statistical Physics"

[5] J.Bekenstein, Lett.Nuovo Cim. 4 (1972) 737; Phys.Rev., D7 (1973) 2339; Phys.Rev., D9 (1974) 3292.

[6] J.M.Bardeen, B.Carter, S.W.Hawking, Comm.Math.Phys., 31 (1973) 161.

[7] S.W.Hawking, Nature 248 (1974) 30; Comm.Math.Phys., 43 (1075) 199. 